\title{
Regular Tooth Brushing is Associated with a Decreased Risk of Metabolic Syndrome According to a Medical Check-Up Database
}

\author{
CHIYO TSUTSUMI*,** AND TATSUYUKI KAKUMA ${ }^{*}$ \\ *Faculty of Nursing, St. Mary's College, Kurume 830-8558, **Kurume University Graduate School of Medicine \\ and ${ }^{\sharp i}$ Biostatistics Center, Kurume University, Kurume 830-0011, Japan \\ Received 12 May 2014, accepted 24 September 2014 \\ J-STAGE advance publication 25 March 2015
}

Edited by YOSHIHIRO FUKUMOTO

\begin{abstract}
Summary: Associations have been reported between periodontal disease and increased cardiovascular disease risk, as well as between healthy self-reported tooth brushing behavior and reduced cardiovascular disease risk. We examined the association between self-reported tooth brushing behavior and the risk of metabolic syndrome (MetS) using a large medical check-up database. A total of 12,548 medical checkup records from a medical screening center in a city in southwest Japan were assessed. Subjects were aged 30 to 59 years. As lifestyle is associated with both tooth brushing and MetS, many lifestyle variables were considered as potential confounders. Logistic regression model were employed with a list of 127 lifestyle variables. Twenty variables associated with both tooth brushing and MetS were selected for both males and females. Furthermore, final confounding variables were selected by principal component analysis to avoid collinearity problems. The association of tooth brushing and MetS was evaluated for males and females separately by calculating adjusted odds ratios (ORs) with selected confounders plus age. The association between daily tooth brushing frequency and MetS risk for both genders was significant after adjusting for confounders, with the risk of MetS decreasing with increased frequency of daily tooth brushing (adjusted OR $=0.57$ (95\% Cl:0.48-0.81), 0.50 (95\% Cl:0.35-0.71), 0.42 (95\% Cl: $0.29-0.61$ ) for males and adjusted $\mathrm{OR}=0.65(95 \% \mathrm{Cl}: 0.48-0.87), 0.44$ (95\% Cl:0.32-0.62) for females). Therefore, oral hygiene, including the frequency of tooth brushing as a significant component, may be an important factor in preventing MetS.
\end{abstract}

Key words tooth brushing, metabolic syndrome, medical checkup database, health behavior, cross-sectional studies, confounding factor

\section{INTRODUCTION}

Physical examinations performed under the auspices of the Japanese national public health insurance system play an important role in secondary preventive care for the purposes of earlier detection and treatment of disease, and it is also one of the national strategies to improve the level of public health in Japan. Selfreporting questionnaires concerning lifestyle, and data from blood tests and body measurements obtained through physical examinations, can be used for the screening of disease and health guidance, and these are accumulated in medical checkup institutes as individual longitudinal data. Analysis of the accumulated data is useful for effective health guidance [1]. As metabolic syndrome (MetS) is a risk factor for cardiovascular disease (CVD) and carries increased risk for CVD [2,3], preventing MetS is of high public health importance. The International Obesity Taskforce has stated that interventions on a sociopolitical level are required to reduce development of MetS in populations [4]. In a previous study the authors conducted an exploratory analysis of health checkup data to research the lifestyle habits that affected the risk of 
MetS. The results suggested that the frequency of tooth brushing, as well as dietary, exercise and drinking habits affected the risk of metabolic syndrome [5]. Although diet, exercise, stress management, smoking and alcohol consumption are identified as items for health guidance in the "Standard Physical Checkup and Health Guidance Program" by the Health, Labor and Welfare Ministry [6], oral care is not included. Yatabe et al. [7] raised a concern about whether it is appropriate to add tooth brushing to the list in the Japan Health Practice Index (JHPI), because although tooth brushing is one of the 10 lifestyle habit behavior items in the JHPI, it correlates weakly with the total score of the JHPI. Although a study by Yoshida evaluated tooth brushing as an active healthy activity, he did not describe its relationship with the results of physical examinations [8]. As described above, although oral care is encouraged as a healthy activity, its effect on MetS has not been clarified.

Over the past two decades, numerous studies have investigated the association of periodontal diseases with an increased risk for CVD [9]. From a public health perspective, improving periodontal health is important because it may reduce the risk of cardiovascular disease [10]. While a meta-analysis [11] of prospective and retrospective studies showed that periodontal disease might only slightly increase the risk of CVD, another meta-analysis study [12] showed an association between systemic bacterial exposure from periodontal disease and coronary heart disease events. However, it has been concluded that properly powered longitudinal case-control and intervention trials are needed to identify how periodontitis and periodontal intervention may impact CVD [13].

In recent years there has been an increasing interest in the relationship between MetS and periodontal disease [14-17]. Although a recent national populationbased study [18] showed that self-reported tooth brushing behavior is inversely associated with CVD, we know of no population-based studies that have examined the association between self-reported tooth brushing behavior and the risk of MetS.

Lifestyle behaviors are considered to be mutually related [5]. Accordingly, the relationship between MetS and oral care activity may possibly be affected by other lifestyle behaviors. Therefore, the purpose of this research was to clarify the relationship between MetS and oral care activity, while controlling for other possible lifestyle behavior effects related to MetS.

\section{MATERIALS AND METHODS}

Data were collected from the periodical medical checkups of 12,548 participants (male: female 7703:4845) from 2004 to 2006 at the medical screening center located in Kurume, Fukuoka Prefecture. Recipients were 30 to 59 years of age (mean \pm SD: $46.3 \pm 7.5$ ), either covered by Social Insurance or National Health Insurance. For those who had multiple checkup data, we utilized data only from their first visit.

\section{High MetS risk group}

The diagnostic criteria of MetS, such as abdominal obesity, lipid metabolic disorder, glucose intolerance and hypertension, were applied to identify the high MetS risk group. Because waist size was not included in the data from the physical examination, visceral fat value was used to show abdominal obesity in place of waist value. In maintaining the diagnostic standard of the National Health and Nutrient Survey 2004 [19], and to obtain more subjects with high MetS risk than by using only one of the diagnostic standards, the high MetS risk conditions were defined by one applicable item being added to abdominal obesity and expressed by binary variables, as shown in Table 1 .

\section{Factors relating to attributes and lifestyle}

The items on the questionnaire form can be broadly classified into three categories: 1) attributes, 2) lifestyle behaviors and 3) mental and emotional factors. The category of attributes had four items, consisting of: absence or presence of persons living together; occupation, working pettern, average of weekly working hours ; the 60 items related to lifestyle behaviors covered the frequency of intake of food and nonessential luxury items, dietary habits, fitness habits, oral health care and the way holidays are spent, and the 64 items related to mental and emotional factors covered amusements, health awareness, and psychological state. All of these were categorical variables other than age.

\section{Analysis of data}

Although five choices ('none', 'once a day', 'twice a day', 'three times a day' and 'more than three times a day') were presented in the tooth-brushing question, some choices were selected very seldom by the subjects. Thus, five items were combined into fewer categories as follows: four categories ('none', 'once a day', 'twice a day' and 'more than twice a day') for males. Since only 4 females answered 'none', the first two categories were combined into 'at most once a day', 
TABLE 1

Criteria used to define high risk MetS

\begin{tabular}{lll}
\hline Obese & & \\
& Body mass percentage & Male \\
Female & $\geq 20 \%$ \\
and/or & $\geq 30 \%$ \\
Body mass index & $\geq 25 \mathrm{~kg} / \mathrm{m}^{2}$ \\
\hline One or more of the following items are added to the above: & \\
Hypertriglyceridemia & $\geq 150 \mathrm{mg} / \mathrm{dl}$ \\
and/or & $<40 \mathrm{mg} / \mathrm{dl}$ \\
Low HDL cholesterol & $\geq 130 \mathrm{mmHg}$ \\
Systolic blood pressure & \\
and/or & $\geq 85 \mathrm{mmHg}$ \\
Diastolic blood pressure & $\geq 110 \mathrm{mg} / \mathrm{dl}$ \\
Fasting plasma glucose &
\end{tabular}

Note* Receiving drug therapy for hypertriglyceridemia, hypertension, or diabetes should be included in the respective items.

resulting in three categories for females.

The purpose of this research was to clarify the relationship between tooth brushing frequency and MetS risk. Generally speaking, when the effect of an exposure on an outcome is examined by an epidemiological study, there may exist a set of covariates affecting both exposure and outcome. These covariates are known to distort the association between exposure and outcome, and are referred to as confounders or confounding variables. Therefore, to properly assess the association, the effect of the confounders must be removed in the analysis. The number of covariates available in this research was 127, and many of the variables were highly associated with each other. Because of this collinearity problem, it was not feasible to adjust for the effects of all 127 covariates in the analysis.

Therefore, a logistic regression model was employed to select variables that were associated with both MetS and tooth brushing frequency. Because lifestyle behaviors were quite different between males and females [5], variable selection was carried out separately by gender. In addition, age was considered as a confounder a priori [5].

The specific method for selecting confounding variables was as follows. First, the strength of association between each covariate and MetS was evaluated using the Wald Chi-Square statistic obtained from a univariate logistic regression model. Because of the large sample size, almost all p-values were highly significant. Since the p-values alone were not useful in the varia- ble selection process, we arbitrarily decided to use the first 50 covariates with the smallest $p$-values as potential confounding variables. The same procedures were used for the tooth brushing categories, except that the univariate logistic regression was replaced with a nominal logistic regression model. Twenty variables that were selected in both procedures were considered confounding variables. Table 2 shows the list of 50 covariates for each gender and the confounding factors were marked with the symbol *.

Although the number of potential confounders was reduced from 127 to 20 for both genders, these 20 variables exhibited strong associations among themselves. To avoid a collinearity problem, further variable selection was necessary. To select a few confounding variables, principal component analysis was carried out. Principal components with an eigenvalue greater than one were retained. The variable with the largest loading score within each principal component was selected as a final confounding variable. Finally, selected confounders plus age were then used in a logistic regression model, and the effect of tooth brushing on MetS risk was evaluated using the ORs.

To evaluate the reliability of our findings, two additional data analyses were performed. The first analysis was to examine the reliability of controlling for the effects of confounding variables. Propensity score stratification is an effective method to adjust for selection bias and confounding in a cross-sectional study [20]. To this end, propensity scores were estimated based 
TABLE 2.

List of 50 covariates for each gender

\begin{tabular}{|c|c|c|c|c|c|c|c|c|c|c|c|}
\hline \multicolumn{6}{|c|}{ Male } & \multicolumn{6}{|c|}{ Female } \\
\hline \multicolumn{3}{|c|}{ MetS risk } & \multicolumn{3}{|c|}{ Tooth brushing } & \multicolumn{3}{|c|}{ MetS risk } & \multicolumn{3}{|c|}{ Tooth brushing } \\
\hline Potential confound & & $\begin{array}{l}\text { Wald- } \\
\text { ChiSq }\end{array}$ & $\begin{array}{l}\text { Potential con- } \\
\text { founder }\end{array}$ & & $\begin{array}{l}\text { Wald- } \\
\text { ChiSq }\end{array}$ & Potential confounc & & $\begin{array}{l}\text { Wald- } \\
\text { ChiSq }\end{array}$ & $\begin{array}{l}\text { Potential con- } \\
\text { founder }\end{array}$ & & $\begin{array}{l}\text { Wald- } \\
\text { ChiSq }\end{array}$ \\
\hline Wakes up early & & 340.1 & $\begin{array}{l}\text { Cannot sleep } \\
\text { because of worry }\end{array}$ & & 221.5 & $\begin{array}{l}\text { Has interest in } \\
\text { losing weight }\end{array}$ & & 70.9 & Yogurt & & 47.1 \\
\hline Inadequate sleep & & 273.9 & $\begin{array}{l}\text { Feels no meaning } \\
\text { to life }\end{array}$ & & 160.0 & Eats too much & $*$ & 50.8 & Sugar in coffee & & 40.7 \\
\hline $\begin{array}{l}\text { Cannot cope with } \\
\text { job }\end{array}$ & & 113.2 & $\begin{array}{l}\text { Lacks concentra- } \\
\text { tion }\end{array}$ & & 157.0 & Eats quickly & $*$ & 48.7 & $\begin{array}{l}\text { Vegetables with } \\
\text { lunch }\end{array}$ & & 39.1 \\
\hline $\begin{array}{l}\text { Burdened by rais- } \\
\text { ing children and } \\
\text { housework }\end{array}$ & & 44.2 & $\begin{array}{l}\text { Feels unmoti- } \\
\text { vated }\end{array}$ & & 137.3 & $\begin{array}{l}\text { Has interest in } \\
\text { walking }\end{array}$ & & 30.6 & $\begin{array}{l}\text { Conscious of salt } \\
\text { intake }\end{array}$ & $*$ & 38.8 \\
\hline $\begin{array}{l}\text { Has chance to } \\
\text { talk with some- } \\
\text { one freely }\end{array}$ & & 31.2 & $\begin{array}{l}\text { Cannot work as } \\
\text { expected }\end{array}$ & & 131.3 & $\begin{array}{l}\text { Drinking fre- } \\
\text { quency }\end{array}$ & & 14.7 & $\begin{array}{l}\text { Favorite season- } \\
\text { ing }\end{array}$ & $*$ & 38.6 \\
\hline $\begin{array}{l}\text { Conscious of } \\
\text { regular life }\end{array}$ & $*$ & 25.7 & $\begin{array}{l}\text { Has no one who } \\
\text { understands their } \\
\text { job }\end{array}$ & & 122.1 & Salty food & & 13.9 & $\begin{array}{l}\text { Too many over- } \\
\text { time hours }\end{array}$ & $*$ & 34.6 \\
\hline Mood swings & & 25.6 & Enjoys daily life & & 102.7 & $\begin{array}{l}\text { Worries about } \\
\text { being sick }\end{array}$ & & 13.5 & Juice & $*$ & 34.4 \\
\hline Working pattern & & 25.3 & $\begin{array}{l}\text { No way to cope } \\
\text { with stress }\end{array}$ & & 101.6 & Irregular diet & & 12.7 & $\begin{array}{l}\text { Green and yellow } \\
\text { vegetables }\end{array}$ & & 34.4 \\
\hline $\begin{array}{l}\text { Too many over- } \\
\text { time hours }\end{array}$ & & 23.7 & $\begin{array}{l}\text { Conscious of } \\
\text { regular life }\end{array}$ & $*$ & 94.2 & $\begin{array}{l}\text { Coffee and red } \\
\text { tea }\end{array}$ & & 12.2 & Hobbies & & 34.2 \\
\hline $\begin{array}{l}\text { Extensive con- } \\
\text { tacts }\end{array}$ & & 19.9 & $\begin{array}{l}\text { Maintains bal- } \\
\text { ance between } \\
\text { activities and rest }\end{array}$ & & 91.8 & $\begin{array}{l}\text { Burdened by } \\
\text { relationship with } \\
\text { neighbors }\end{array}$ & $*$ & 12.2 & Fruits & & 26.1 \\
\hline $\begin{array}{l}\text { Assumes heavy } \\
\text { responsibility }\end{array}$ & & 17.0 & Occupation & & 91.4 & Fried vegetables & & 11.6 & Fatty meat & & 25.0 \\
\hline Walking & & 17.0 & $\begin{array}{l}\text { Has interest in } \\
\text { walking }\end{array}$ & $*$ & 87.1 & $\begin{array}{l}\text { Seasonings on } \\
\text { the table }\end{array}$ & $*$ & 10.3 & $\begin{array}{l}\text { Problems with } \\
\text { boss and col- } \\
\text { leagues }\end{array}$ & & 24.5 \\
\hline $\begin{array}{l}\text { Has interest in } \\
\text { walking }\end{array}$ & $*$ & 16.9 & $\begin{array}{l}\text { Exercise other } \\
\text { than walking }\end{array}$ & $*$ & 85.6 & $\begin{array}{l}\text { Favorite season- } \\
\text { ing }\end{array}$ & & 9.9 & Soy beans & & 24.4 \\
\hline $\begin{array}{l}\text { Exercise other } \\
\text { than walking }\end{array}$ & $*$ & 16.9 & $\begin{array}{l}\text { Has interest in } \\
\text { exercise }\end{array}$ & & 85.0 & $\begin{array}{l}\text { Suffers from } \\
\text { gastrointestinal } \\
\text { upset }\end{array}$ & & 9.8 & $\begin{array}{l}\text { Has interest in } \\
\text { exercise }\end{array}$ & & 23.4 \\
\hline $\begin{array}{l}\text { Exercises during } \\
\text { holidays }\end{array}$ & $*$ & 16.8 & $\begin{array}{l}\text { Exercises during } \\
\text { holidays }\end{array}$ & $*$ & 81.9 & $\begin{array}{l}\text { Too many over- } \\
\text { time hours }\end{array}$ & $*$ & 9.3 & $\begin{array}{l}\text { Vegetables for } \\
\text { breakfast }\end{array}$ & $*$ & 20.9 \\
\hline $\begin{array}{l}\text { Does housekeep- } \\
\text { ing during holi- } \\
\text { days }\end{array}$ & $*$ & 14.6 & $\begin{array}{l}\text { Does housekeep- } \\
\text { ing during holi- } \\
\text { days }\end{array}$ & $*$ & 78.3 & $\begin{array}{l}\text { Sometimes skips } \\
\text { meals }\end{array}$ & $*$ & 9.2 & $\begin{array}{l}\text { Eats more for } \\
\text { dinner }\end{array}$ & & 20.2 \\
\hline $\begin{array}{l}\text { Rests at home } \\
\text { during holidays }\end{array}$ & $*$ & 14.2 & $\begin{array}{l}\text { Rests at home } \\
\text { during holidays }\end{array}$ & $*$ & 73.6 & $\begin{array}{l}\text { Vegetables for } \\
\text { dinner }\end{array}$ & $*$ & 8.3 & $\begin{array}{l}\text { Sometimes skips } \\
\text { meals }\end{array}$ & $*$ & 18.9 \\
\hline Enjoys drinking & & 14.1 & $\begin{array}{l}\text { Enjoys playing } \\
\text { sports }\end{array}$ & $*$ & 71.9 & $\begin{array}{l}\text { Extensive con- } \\
\text { tacts }\end{array}$ & & 8.3 & Smokes & & 17.3 \\
\hline Enjoys eating & & 14.1 & Hobbies & & 69.3 & $\begin{array}{l}\text { Thinks current } \\
\text { job is not suitable }\end{array}$ & & 8.3 & $\begin{array}{l}\text { Hobbies or } \\
\text { learning during } \\
\text { holidays }\end{array}$ & & 17.0 \\
\hline $\begin{array}{l}\text { Enjoys playing s } \\
\text { ports }\end{array}$ & $*$ & 13.4 & $\begin{array}{l}\text { Has interest in } \\
\text { losing weight }\end{array}$ & $*$ & 65.3 & Cake & & 7.9 & $\begin{array}{l}\text { Seasonings on } \\
\text { the table }\end{array}$ & $*$ & 16.9 \\
\hline $\begin{array}{l}\text { Has interest in } \\
\text { losing weight }\end{array}$ & $*$ & 13.4 & Smoking & & 60.8 & Wakes up early & & 7.7 & Seaweed & & 16.3 \\
\hline
\end{tabular}




\begin{tabular}{|c|c|c|c|c|c|c|c|c|c|c|c|}
\hline Fatty meat & $*$ & 13.1 & Potatoes & & 55.6 & $\begin{array}{l}\text { Conscious of salt } \\
\text { intake }\end{array}$ & $*$ & 7.0 & $\begin{array}{l}\text { Maintains bal- } \\
\text { ance between } \\
\text { activities and rest }\end{array}$ & & 15.7 \\
\hline Egg & & 12.8 & Fatty meat & $*$ & 50.0 & Drinking period & & 6.9 & Noodle soup & & 15.3 \\
\hline Yogurt & $*$ & 12.2 & Soy beans & & 45.8 & Eats dinner & & 6.8 & $\begin{array}{l}\text { No particular } \\
\text { method to cope } \\
\text { with stress }\end{array}$ & $*$ & 14.6 \\
\hline Fried vegetables & & 11.9 & Milk & & 38.4 & $\begin{array}{l}\text { Difficulty falling } \\
\text { asleep }\end{array}$ & & 6.4 & $\begin{array}{l}\text { Burdened by } \\
\text { relationship with } \\
\text { neighbors }\end{array}$ & & 14.4 \\
\hline Deep fried food & & 11.3 & Yogurt & $*$ & 35.7 & Enjoys drinking & & 5.8 & $\begin{array}{l}\text { Heavy responsi- } \\
\text { bility at work }\end{array}$ & & 14.3 \\
\hline Fatty staple food & & 11.3 & Seaweed & & 35.5 & Worries about job & $*$ & 5.4 & Processed food & * & 12.8 \\
\hline $\begin{array}{l}\text { Vegetables for } \\
\text { breakfast }\end{array}$ & $*$ & 11.1 & $\begin{array}{l}\text { Vegetables for } \\
\text { breakfast }\end{array}$ & $*$ & 30.7 & Japanese cake & & 5.3 & $\begin{array}{l}\text { Vegetables for } \\
\text { dinner }\end{array}$ & $*$ & 12.5 \\
\hline $\begin{array}{l}\text { Green and yellow } \\
\text { vegetables }\end{array}$ & * & 10.4 & $\begin{array}{l}\text { Vegetables for } \\
\text { lunch }\end{array}$ & & 30.1 & $\begin{array}{l}\text { Has own counter- } \\
\text { measures }\end{array}$ & & 5.2 & Eats too much & * & 11.8 \\
\hline $\begin{array}{l}\text { Dishes cooked } \\
\text { with sugar and } \\
\text { sweet cooking } \\
\text { rice wine }\end{array}$ & & 10.0 & $\begin{array}{l}\text { Vegetables for } \\
\text { dinner }\end{array}$ & & 30.1 & $\begin{array}{l}\text { Preparation of } \\
\text { dinner }\end{array}$ & $*$ & 5.2 & $\begin{array}{l}\text { Often eats a la } \\
\text { carte when eating } \\
\text { out }\end{array}$ & & 11.2 \\
\hline Sugar in coffee & $*$ & 9.7 & $\begin{array}{l}\text { Green and yellow } \\
\text { vegetables }\end{array}$ & $*$ & 29.1 & Working pattern & & 5.0 & Soup dish & & 10.9 \\
\hline Juice & $*$ & 9.1 & Fruits & & 27.3 & $\begin{array}{l}\text { Vegetables for } \\
\text { breakfast }\end{array}$ & $*$ & 4.9 & Eats quickly & $*$ & 10.6 \\
\hline Cake & & 8.9 & Sugar in coffee & $*$ & 26.2 & $\begin{array}{l}\text { Pickles and food } \\
\text { boiled in soy } \\
\text { sauce }\end{array}$ & $*$ & 4.9 & Seasoned rice & & 10.5 \\
\hline Drinking period & & 7.9 & Juice & $*$ & 26.0 & $\begin{array}{l}\text { Cannot cope with } \\
\text { job }\end{array}$ & & 4.9 & $\begin{array}{l}\text { Too many over- } \\
\text { time hours }\end{array}$ & & 10.4 \\
\hline Eats quickly & & 7.5 & Irregular diet & & 21.7 & $\begin{array}{l}\text { Burdened by rais- } \\
\text { ing children and } \\
\text { housework }\end{array}$ & & 4.8 & $\begin{array}{l}\text { Solves problems } \\
\text { immediately }\end{array}$ & $*$ & 10.2 \\
\hline Eats too much & & 7.4 & Late dinner & & 21.5 & $\begin{array}{l}\text { Exercises during } \\
\text { holidays }\end{array}$ & $*$ & 4.8 & $\begin{array}{l}\text { Pickles and food } \\
\text { boiled in soy } \\
\text { sauce }\end{array}$ & $*$ & 10.2 \\
\hline $\begin{array}{l}\text { Eats out more } \\
\text { than } 2 \text { times a } \\
\text { day }\end{array}$ & & 7.3 & $\begin{array}{l}\text { Sometimes skips } \\
\text { meals }\end{array}$ & & 21.1 & $\begin{array}{l}\text { No particular } \\
\text { method to cope } \\
\text { with stress }\end{array}$ & $*$ & 4.6 & Mood swings & & 10.1 \\
\hline $\begin{array}{l}\text { Often eats a la } \\
\text { carte when eating } \\
\text { out }\end{array}$ & $*$ & 7.2 & Eats dinner & & 21.0 & Late dinner & & 4.3 & $\begin{array}{l}\text { Exercises during } \\
\text { holidays }\end{array}$ & * & 10.0 \\
\hline $\begin{array}{l}\text { Has likes and } \\
\text { dislikes }\end{array}$ & $*$ & 7.0 & $\begin{array}{l}\text { Often eats a la } \\
\text { carte when eating } \\
\text { out }\end{array}$ & $*$ & 21.0 & $\begin{array}{l}\text { Box lunch from } \\
\text { the market }\end{array}$ & & 4.2 & Deep fried food & & 9.8 \\
\hline $\begin{array}{l}\text { Favorite season- } \\
\text { ing }\end{array}$ & $*$ & 6.9 & $\begin{array}{l}\text { Has likes and } \\
\text { dislikes }\end{array}$ & $*$ & 20.9 & $\begin{array}{l}\text { Does volunteer } \\
\text { work during } \\
\text { holidays }\end{array}$ & & 4.2 & $\begin{array}{l}\text { Assumes heavy } \\
\text { responsibility }\end{array}$ & & 9.5 \\
\hline $\begin{array}{l}\text { Conscious of salt } \\
\text { intake }\end{array}$ & $*$ & 6.9 & $\begin{array}{l}\text { Favorite season- } \\
\text { ing }\end{array}$ & $*$ & 20.6 & $\begin{array}{l}\text { Solves problems } \\
\text { immediately }\end{array}$ & $*$ & 4.0 & $\begin{array}{l}\text { Feels unmoti- } \\
\text { vated }\end{array}$ & & 9.3 \\
\hline Soup & $*$ & 6.8 & $\begin{array}{l}\text { Conscious of salt } \\
\text { intake }\end{array}$ & $*$ & 20.0 & $\begin{array}{l}\text { Consults with } \\
\text { expert or hospital }\end{array}$ & & 4.0 & Milk & & 9.3 \\
\hline Seasoned rice & & 6.5 & $\begin{array}{l}\text { Seasonings on } \\
\text { the table }\end{array}$ & & 19.8 & $\begin{array}{l}\text { Worries about } \\
\text { family }\end{array}$ & & 4.0 & $\begin{array}{l}\text { Preparation of } \\
\text { dinner }\end{array}$ & * & 8.7 \\
\hline Salty food & $*$ & 6.5 & Soup & $*$ & 19.6 & $\begin{array}{l}\text { Worries about } \\
\text { small matters }\end{array}$ & & 3.9 & Enjoys holidays & & 8.6 \\
\hline Processed food & & 6.1 & Noodle soup & & 19.1 & Juice & $*$ & 3.8 & Worries about job & $*$ & 8.4 \\
\hline
\end{tabular}




\begin{tabular}{|c|c|c|c|c|c|c|c|c|c|c|}
\hline $\begin{array}{l}\text { Pickles and food } \\
\text { boiled in soy } \\
\text { sauce }\end{array}$ & 6.0 & Salty food & * & 19.0 & $\begin{array}{l}\text { Enjoys playing } \\
\text { sports }\end{array}$ & $*$ & 3.7 & Busy & & 8.4 \\
\hline $\begin{array}{l}\text { Suffers from } \\
\text { headaches }\end{array}$ & 5.8 & $\begin{array}{l}\text { Boxed lunch } \\
\text { from the market }\end{array}$ & & 15.0 & Processed food & $*$ & 3.7 & $\begin{array}{l}\text { Eats out more } \\
\text { than } 2 \text { times a } \\
\text { day }\end{array}$ & & 8.2 \\
\hline $\begin{array}{l}\text { Suffers from } \\
\text { gastrointestinal } \\
\text { upset }\end{array}$ & 5.7 & $\begin{array}{l}\text { Prepares break- } \\
\text { fast }\end{array}$ & & 14.3 & Potatoes & & 3.6 & $\begin{array}{l}\text { Exercise other } \\
\text { than walking }\end{array}$ & & 8.1 \\
\hline Feels lethargic & 5.7 & $\begin{array}{l}\text { Fatigue cannot be } \\
\text { overcome by } \\
\text { sleep }\end{array}$ & & 14.0 & Skim milk & & 3.2 & $\begin{array}{l}\text { Does housekeep- } \\
\text { ing during holi- } \\
\text { days }\end{array}$ & $*$ & 7.9 \\
\hline $\begin{array}{l}\text { Worries about } \\
\text { being sick }\end{array}$ & 5.5 & Feels lazy & & 13.2 & $\begin{array}{l}\text { Does housekeep- } \\
\text { ing during holi- } \\
\text { days }\end{array}$ & $*$ & 3.0 & $\begin{array}{l}\text { Enjoys playing } \\
\text { sports }\end{array}$ & * & 7.6 \\
\hline
\end{tabular}

*: Treated as confounder.

on logistic regression models where a binary tooth brushing frequency variable was defined as "once a day or less" or "more than once a day" and was used as the response variable with a set of potential confounders as explanatory variables in the model. The MantelHaenszel common ORs were obtained based on five strata constructed from the ranks of the propensity scores. Because statistical tests were based on a relatively large sample size in our study, a second analysis examined the effect of sample size. Five thousand bootstrap samples were generated, together with the empirical bootstrap distribution of the Mantel-Haenszel common ORs based on the propensity score method. Empirical 95\% confidence intervals of ORs for males and females were constructed. Statistical Analysis System 9.1 software was used in all statistical analyses.

\section{Ethics approval}

This epidemiologic study used medical examination data for which informed consent was obtained based on the "Ethics Guidelines for Epidemiological Research" [21]. We obtained approval for the study from the Ethics Committee of the college and the hospital affiliated with the medical screening center [2006 Jun 14]. We obtained data that excluded full names and dates of birth, and set up anonymity safeguards that made it impossible for individuals to be identified.

\section{RESULTS}

\section{Characteristics of the subjects}

The prevalence of MetS risk was $41 \%$ in males and $10 \%$ in females. These percentages were slightly higher than the $36.2 \%$ in nationally surveyed males and $10 \%$ in nationally surveyed females [19]. The tooth brushing frequencies were: $2.1 \%$ less than once a day, $35.9 \%$ once a day, $46.8 \%$ twice a day and $15.3 \%$ more than twice a day in males; and $10.0 \%$ less than once a day, $53.4 \%$ twice a day and $36.5 \%$ more than twice a day in females. Table 3 shows the distribution of the tooth brushing frequencies divided into two groups, with once a day or less and more than once per day, and MetS risk according to gender, age group and occupation. Regarding occupational status, White Collar was defined as including professional technical work, managerial and clerical work, and Worker was defined as including all other occupations.

\section{Odds ratios for tooth brushing and MetS risk}

The association between MetS risk (high vs. low) and categorized tooth brushing behavior was evaluated using the logistic regression models. The adjusted ORs are shown in Table 4 for males and females. For males, the odds for high MetS risk among people who do not brush their teeth were used as a reference. The ORs were $0.57(0.4-0.81), 0.50(0.35-0.71)$ and 0.42 (0.29-0.61) among the once a day, twice a day and more than twice a day groups, respectively. For females, the odds for high MetS risk among people who brush their teeth at most once a day were used as a reference. The ORs were $0.65(0.48-0.87)$ and $0.44(0.32-0.62)$ for the twice a day and more than twice a day groups, respectively. Frequency of tooth brushing may have a dose-response effect on risk of MetS as suggested by the magnitude of the ORs.

The Mantel-Haenszel common ORs based on the propensity score methods were $(0.86,0.97)$ and $(0.50$, 0.81 ) for males and females, respectively. Empirical 
TABLE 3

Distribution of tooth brushing frequency and MetS risk based upon selected confounding variables

\begin{tabular}{|c|c|c|c|c|c|c|c|}
\hline & & \multicolumn{3}{|c|}{ MetS Risk } & \multicolumn{3}{|c|}{ Tooth brushing } \\
\hline & & Low & High & Total & More than once & Once or less & Total \\
\hline \multicolumn{8}{|l|}{ Attribute } \\
\hline \multirow[t]{2}{*}{ Gender } & Male & $4553(59.1 \%)$ & $3150(40.9 \%)$ & 7703 & $4777(62.1 \%)$ & $2921(37.9 \%)$ & 7698 \\
\hline & Female & $4371(90.2 \%)$ & $474(9.8 \%)$ & 4845 & $4342(90.0 \%)$ & $485(10.0 \%)$ & 4827 \\
\hline \multirow[t]{3}{*}{ Age (years) } & $30-39$ & $2024(76.7 \%)$ & $615(23.3 \%)$ & 2639 & $2015(76.4 \%)$ & $624(23.6 \%)$ & 2639 \\
\hline & $40-49$ & $3677(72.0 \%)$ & $1431(28.0 \%)$ & 5108 & $3757(73.6 \%)$ & $1350(26.4 \%)$ & 5107 \\
\hline & $50-59$ & $3223(67.1 \%)$ & $1578(32.9 \%)$ & 4801 & $3347(70.0 \%)$ & $1432(30.0 \%)$ & 4779 \\
\hline \multicolumn{8}{|c|}{ Selected Confounders } \\
\hline \multicolumn{8}{|l|}{ Male } \\
\hline \multirow{3}{*}{$\begin{array}{l}\text { Spends } \\
\text { holidays } \\
\text { exercising }\end{array}$} & No & $3365(57.9 \%)$ & $2445(42.1 \%)$ & 5810 & $3482(59.9 \%)$ & $2328(40.1 \%)$ & 5810 \\
\hline & Yes & $1186(62.8 \%)$ & $702(37.2 \%)$ & 1888 & $1295(68.6 \%)$ & $593(31.4 \%)$ & 1888 \\
\hline & $\begin{array}{l}\text { Prefers plain } \\
\text { taste }\end{array}$ & $1250(61.5 \%)$ & $782(38.5 \%)$ & 2032 & $1374(67.6 \%)$ & $658(32.4 \%)$ & 2032 \\
\hline \multirow{2}{*}{$\begin{array}{l}\text { Favorite } \\
\text { seasoning }\end{array}$} & Normal & $2740(58.9 \%)$ & $1914(41.1 \%)$ & 4654 & $2835(60.9 \%)$ & $1819(39.1 \%)$ & 4654 \\
\hline & $\begin{array}{l}\text { Prefers strong } \\
\text { taste }\end{array}$ & $532(55.4 \%)$ & $429(44.6 \%)$ & 961 & $537(55.9 \%)$ & $424(44.1 \%)$ & 961 \\
\hline \multirow{4}{*}{ Eats soup } & None & $470(62.8 \%)$ & $279(37.2 \%)$ & 749 & $401(53.5 \%)$ & $348(46.5 \%)$ & 749 \\
\hline & 1 bowl a day & $3192(59.5 \%)$ & $2175(40.5 \%)$ & 5367 & $3341(62.3 \%)$ & $2026(37.7 \%)$ & 5367 \\
\hline & 2 bowls a day & $800(56.0 \%)$ & $628(44.0 \%)$ & 1428 & $942(66.0 \%)$ & $486(34.0 \%)$ & 1428 \\
\hline & $\begin{array}{l}3 \text { bowls or } \\
\text { more a day }\end{array}$ & $60(58.3 \%)$ & $43(41.7 \%)$ & 103 & $62(60.2 \%)$ & $41(39.8 \%)$ & 103 \\
\hline \multirow{4}{*}{$\begin{array}{l}\text { Sugar in } \\
\text { coffee }\end{array}$} & $\begin{array}{l}\text { Does not } \\
\text { drink }\end{array}$ & $634(57.5 \%)$ & $469(42.5 \%)$ & 1103 & $700(63.5 \%)$ & $403(36.5 \%)$ & 1103 \\
\hline & No sugar & $2681(57.3 \%)$ & $2000(42.7 \%)$ & 4681 & $3050(65.2 \%)$ & $1631(34.8 \%)$ & 4681 \\
\hline & 1 teaspoon & $842(63.6 \%)$ & $482(36.4 \%)$ & 1324 & $740(55.9 \%)$ & $584(44.1 \%)$ & 1324 \\
\hline & 2 teaspoons & $358(67.3 \%)$ & $174(32.7 \%)$ & 532 & $252(47.4 \%)$ & $280(52.6 \%)$ & 532 \\
\hline \multirow{4}{*}{$\begin{array}{l}\text { Has interest } \\
\text { in losing } \\
\text { weight }\end{array}$} & No interest & $1587(83.2 \%)$ & $321(16.8 \%)$ & 1908 & $1138(59.6 \%)$ & $770(40.4 \%)$ & 1908 \\
\hline & $\begin{array}{l}\text { Interested, } \\
\text { but does not } \\
\text { intend to lose } \\
\text { weight soon }\end{array}$ & $940(55.9 \%)$ & $742(44.1 \%)$ & 1682 & $982(58.4 \%)$ & $700(41.6 \%)$ & 1682 \\
\hline & $\begin{array}{l}\text { Interested and } \\
\text { wants to start } \\
\text { immediately }\end{array}$ & $1257(44.0 \%)$ & $1602(56.0 \%)$ & 2859 & $1783(62.4 \%)$ & $1076(37.6 \%)$ & 2859 \\
\hline & $\begin{array}{l}\text { Interested and } \\
\text { has started }\end{array}$ & $767(61.4 \%)$ & $482(38.6 \%)$ & 1249 & $874(70.0 \%)$ & $375(30.0 \%)$ & 1249 \\
\hline \multirow{2}{*}{$\begin{array}{l}\text { Spends } \\
\text { holidays } \\
\text { housekeeping }\end{array}$} & No & $3756(58.5 \%)$ & $2669(41.5 \%)$ & 6425 & $3937(61.3 \%)$ & $2488(38.7 \%)$ & 6425 \\
\hline & Yes & $795(62.5 \%)$ & $478(37.5 \%)$ & 1273 & $840(66.0 \%)$ & $433(34.0 \%)$ & 1273 \\
\hline
\end{tabular}




\begin{tabular}{|c|c|c|c|c|c|c|c|c|c|}
\hline \multirow[t]{3}{*}{$\begin{array}{l}\text { Favorite } \\
\text { seasoning }\end{array}$} & $\begin{array}{l}\text { Prefers plain } \\
\text { taste }\end{array}$ & $1767(91.7 \%)$ & 160 & $(8.3 \%)$ & 1927 & $1769(91.8 \%)$ & 158 & $(8.2 \%)$ & 1927 \\
\hline & Normal & $2357(89.4 \%)$ & 279 & $(10.6 \%)$ & 2636 & $2344(88.9 \%)$ & 292 & $(11.1 \%)$ & 2636 \\
\hline & $\begin{array}{l}\text { Prefers strong } \\
\text { taste }\end{array}$ & $210(86.8 \%)$ & 32 & $(13.2 \%)$ & 242 & $208(86.0 \%)$ & 34 & $(14.0 \%)$ & 242 \\
\hline \multirow{2}{*}{$\begin{array}{l}\text { Worries about } \\
\text { job }\end{array}$} & No & $1191(88.7 \%)$ & 151 & $(11.3 \%)$ & 1342 & $1196(89.1 \%)$ & 146 & $(10.9 \%)$ & 1342 \\
\hline & Yes & $2860(91.0 \%)$ & 283 & $(9.0 \%)$ & 3143 & $2838(90.3 \%)$ & 305 & $(9.7 \%)$ & 3143 \\
\hline \multirow{3}{*}{$\begin{array}{l}\text { Pickles and } \\
\text { food boiled in } \\
\text { soy sauce }\end{array}$} & Does not eat & $1360(91.5 \%)$ & 127 & $(8.5 \%)$ & 1487 & $1353(91.0 \%)$ & 134 & $(9.0 \%)$ & 1487 \\
\hline & Once a day & $2488(90.0 \%)$ & 275 & $(10.0 \%)$ & 2763 & $2465(89.2 \%)$ & 298 & $(10.8 \%)$ & 2763 \\
\hline & Twice a day & $400(88.1 \%)$ & 54 & $(11.9 \%)$ & 454 & $417(91.9 \%)$ & 37 & $(8.1 \%)$ & 454 \\
\hline \multirow{2}{*}{$\begin{array}{l}\text { Spends } \\
\text { holidays } \\
\text { exercising }\end{array}$} & No & $3977(90.0 \%)$ & 444 & $(10.0 \%)$ & 4421 & $3968(89.8 \%)$ & 453 & $(10.2 \%)$ & 4421 \\
\hline & Yes & $379(93.3 \%)$ & 27 & $(6.7 \%)$ & 406 & $374(92.1 \%)$ & 32 & $(7.9 \%)$ & 406 \\
\hline \multirow{2}{*}{ Eats quickly } & No & $1974(93.6 \%)$ & 134 & $(6.4 \%)$ & 2108 & $1926(91.4 \%)$ & 182 & $(8.6 \%)$ & 2108 \\
\hline & Yes & $2360(87.5 \%)$ & 337 & $(12.5 \%)$ & 2697 & $2395(88.8 \%)$ & 302 & $(11.2 \%)$ & 2697 \\
\hline \multirow{3}{*}{$\begin{array}{l}\text { Preparation } \\
\text { of dinner }\end{array}$} & $\begin{array}{l}\text { Prepares } \\
\text { alone }\end{array}$ & $3395(89.5 \%)$ & 397 & $(10.5 \%)$ & 3792 & $3405(89.8 \%)$ & 387 & $(10.2 \%)$ & 3792 \\
\hline & $\begin{array}{l}\text { Prepared by } \\
\text { family }\end{array}$ & $852(93.3 \%)$ & 61 & $(6.7 \%)$ & 913 & $832(91.1 \%)$ & 81 & $(8.9 \%)$ & 913 \\
\hline & $\begin{array}{l}\text { Eats out or } \\
\text { other }\end{array}$ & $87(87.0 \%)$ & 13 & $(13.0 \%)$ & 100 & $84(84.0 \%)$ & 16 & $(16.0 \%)$ & 100 \\
\hline \multirow{2}{*}{$\begin{array}{l}\text { Solves prob- } \\
\text { lems immedi- } \\
\text { ately }\end{array}$} & No & $3257(90.7 \%)$ & 333 & $(9.3 \%)$ & 3590 & $3206(89.6 \%)$ & 373 & $(10.4 \%)$ & 3579 \\
\hline & Yes & $1114(88.8 \%)$ & 141 & $(11.2 \%)$ & 1255 & $1136(91.0 \%)$ & 112 & $(9.0 \%)$ & 1248 \\
\hline
\end{tabular}

95\% confidence intervals of ORs based on the bootstrap methods were $(0.86,0.97)$ and $(0.51,0.99)$ for males and females, respectively. Results from two additional data analyses suggest that our findings did not depend on the methods of adjusting confounding bias nor the large sample size.

\section{DISCUSSION}

The prevalence of MetS, including among the high-risk group subjected to this research, was similar to that of the national survey. It has been shown that the distribution of tooth brushing frequency and MetS risk are very similar by gender, age group and occupation, which suggests that the correlation between them is strong. López et al. has found that there may be a correlation between coronary heart disease and periodontal disease using a case-control study, and considers that they may be in the same cluster [22]. This sug- gests a correlation within a group having the same covariate or lifestyle. However, the results of the present study clarified that the significant correlation that is found after the adjustment for common lifestyle factors cannot be fully explained only because they are in the same cluster. A prospective cohort study in Japan reported that the MetS components (positive conversions of blood pressure and the blood-lipid index) became positive four years after the formation of a periodontal pocket, which supports our findings [16]. One explanation is that a protein component called inflammatory cytokine, produced by the body's own cells, and its stimulation caused by endotoxin derived from periodontal disease, is carried throughout the body, causing the onset and progression of a systemic illness. In the experimental intervention trial whereby subjects refrained from all oral hygiene measures, including the use of dentifrices, mouth rinses, dental floss, and chewing gum, $56 \%$ of participants showed an increase 
TABLE 4.

Odd ratios for MetS risk and tooth brushing adjusted for age and confounding variables

\begin{tabular}{cccccccc}
\hline \multicolumn{7}{c}{ Male } & \multicolumn{5}{c}{ Female } \\
\hline $\begin{array}{c}\text { Tooth brushing } \\
\text { frequency }\end{array}$ & Odds ratio* & $95 \% \mathrm{CI}$ & P value & $\begin{array}{c}\text { Tooth brushing } \\
\text { frequency }\end{array}$ & $\begin{array}{c}\text { Odds } \\
\text { ratio** }\end{array}$ & $95 \% \mathrm{CI}$ & P value \\
\hline Non & - & & - & At most once a day & & \\
Once a day & 0.57 & $0.40-0.81$ & $<0.001$ & Twice a day & 0.65 & $0.48-0.87$ & 0.004 \\
Twice a day & 0.50 & $0.35-0.71$ & $<0.001$ & More than twice a day & 0.44 & $0.32-0.62$ & $<0.001$ \\
More than twice a day & 0.42 & $0.29-0.61$ & $<0.001$ & & & & \\
\hline
\end{tabular}

Adjusted Factors were "Age”, "Exercises during holidays", "Favorite seasoning”, "Eats soup”, "Sugar in coffee”, "Has interest in losing weight " and "Does housekeeping during holidays" for males

Adjusted Factors were "Age", "Favorite seasoning", "Worries about job", "Sugar in coffee", "Pickles and food boiled in soy sauce", "Exercises during holidays", "Eats quickly", "Preparation of dinner" and "Solves problems immediately" for females

in blood endotoxin levels [23]. This suggests that oral care may be a significant factor in preventing MetS. Meanwhile, tooth brushing is an important component of oral care, and our findings of a dose-response relationship between tooth brushing and MetS risk calls for further research in this field.

\section{LIMITATIONS}

Several limitations in this study ought to be mentioned. The first limitation is the lack of generalizability of our findings because of possible selection bias as a result of our limited study population. Our findings were largely based on self-reported tooth brushing behavior. These responses were obtained from a health questionnaire, which may lack reliability and reproducibility. The third limitation is a lack of dental examination data, such as probing pocket depth and tooth loss. Although a statistically significant association between tooth brushing behavior and risk of MetS is tenable, a carefully designed randomized controlled intervention study is required to establish a causal relationship between oral care activities and MetS risk. Although there are many studies that support the value of a healthy lifestyle, some debate exists on the effectiveness of interventions to reduce MetS. To this end, the authors are engaged in an intervention study to evaluate the effect of oral care activities on other lifestyle factors and MetS risk.

\section{CONCLUSION}

Our analyses suggest that self-reported oral care activities are associated with increased risk of MetS after controlling for the effects of many confounding lifestyle factors. This finding may be used to enhance guid- ance that is provided by health care professionals to participants of medical check-ups. Additionally, we demonstrate the practicality of using statistical methods to analyze large medical databases to develop evidence-based health care strategies.

ACKNOWLEDGMENTS: This work was supported by a grant from the Ministry of Education, Culture, Sports, Science and Technology (No. 21659541). Conflicts of Interest: The authors have no conflicts of interest to declare.

\section{REFERENCES}

1. Yanagawa $H$. The use of computer and collection of information. Nursing Association, editor. Occupational Manual for Public Health Nurse. 9th ed. Tokyo. Japanese Nursing Association; 1999. p. 503. (in Japanese)

2. Wilson PWF, D'Agostino RB, Parise H, Sullivan L, and Meigs JB. Metabolic syndrome as a precursor of cardiovascular disease and type 2 diabetes mellitus. Circulation 2005; 112:3066-3072.

3. Eckel RH, Grundy SM, and Zimmet PZ. The metabolic syndrome. Lancet 2005; 365:1415-1428.

4. James PT, Rigby N, and Leach R. International Obesity Task Force. The obesity epidemic, metabolic syndrome and future prevention strategies. Eur J Cardiovasc Prev Rehabil 2004; 11:3-8.

5. Tsutsumi C. Profiling metabolic syndrome from routine physical examinations based on health behavior items on a life-style questionnaire: application of tree-based methods. J Jpn Acad Nurs Sci 2009; 29:21-28.

6. Ministry of Health, Labour and Welfare. Standard Physical Checkup and Health Guidance Program [Internet]. 2007 [cited 2014 Mar 1]. Available from:http://www.mhlw.go.jp/ stf/seisakunitsuite/bunya/kenkou_iryou/kenkou/seikatsu/ link-list.html

7. Yatabe H, Sugimori H, Suka M, Iida Y, Nakamura T et al. Development of the New Assessment Tools for Lifestyles: Japanese Health Practice Index (JHPI). JMHTS. 2001; 28:302-310. 
8. Yoshida K. Lifestyle disease prevention 2. Tokyo; 2000. Chapter 2, Doctor's questions for lifestyle habit compiled by Health Assessment Exploratory Committee and Health Assessment Manual for the Prevention of Lifestyle Disease and Need for Long-term Care by Health Science Research; p. 23-28.

9. Dumitrescu AL, and Inagaki K. Interrelationships between periodontal disease and mortality, cardiovascular disease, MS, diabetes mellitus. In: Dumitrescu AL, editor. Etiology and pathogenesis of periodontal disease. Berlin Heidelberg: Springer-Verlag; 2010. p. 125-157.

10. Oral health in America: A report of the Surgeon General: U. S. Department of Health and Human Services, National Institute of Dental and Craniofacial Research, National Institutes of Health [Internet]. 2000 [cited 2014 Mar 1]. Available from: http://www2.nidcr.nih.gov/sgr/sgrohweb/ welcome.htm

11. Behekar AA, Singh S, Sara S, Molnar J, and Arora R. The prevalence and incidence of coronary heart disease is significantly increased in periodontitis: a meta-analysis. Am Heart J 2007; 154:830-837.

12. Mustapha IZ, Debrey S, Oladubu M, and Ugarte R. Markers of systemic bacterial exposure in periodontal disease and cardiovascular disease risk: a systematic review and metaanalysis. J Periodontol 2007; 78:289-302.

13. Persson GR, and Persson RE. Cardiovascular disease and periodontitis: an update on the associations and risk. J Clin Periodontol 2008; 35:362-379.

14. Shimazaki Y, Saito T, Kiyohara Y, Kato I, Kubo M et al. Relationship between electrocardiographic abnormalities and periodontal disease: the Hisayama Study. J Periodontol 2004; 75:791-797.

15. Khader Y, Khassawneh B, Obeidat B, Hammad M,
El-Salem K et al. Periodontal status of patients with metabolic syndrome compared with those without metabolic syndrome. J Periodontol 2008; 79:2048-2053.

16. Morita T, Yamazaki Y, Mita A, Takada K, Seto M et al. A cohort study on the association between periodontal disease and the development of metabolic syndrome. J Periodontol 2010; 81:512-519.

17. Bensly L, Vanenwyk J, and Ossiander EM. Associations of self-reported periodontal disease with metabolic syndrome and number of self-reported chronic conditions. Prev Chronic Dis 2011; 8:A50.

18. Oliveira C, Watt R, and Hamer M. Toothbrushing, inflammation, and risk of cardiovascular disease: results from Scottish Health Survey. BMJ 2010; 340:c2451.

19. Ministry of Health, Labour and Welfare. The National Health and Nutrient Survey [Internet]. 2004 [cited 2014 Mar 1]. Available from: http://www.mhlw.go.jp/bunya/kenkou/kenkou_eiyou_chousa.html

20. Faries DE, Leon AC, Haro JM, and Ogenchain RL. Analysis of observational health care data using SAS. Cary, NC, USA: SAS Institute Inc.; 2010.

21. Ministry of Education, Culture, Sports, Science and Technology, Ministry of Health, Labour and Welfare. Ethical Guidelines for Epidemiological Research [Internet]. 2002 June 17 [cited 2014 Mar 1]. Available from:http://www. lifescience.mext.go.jp/files/pdf/n796_01.pdf

22. López R, Oyarzún M, Naranjo C, Cumsille F, Ortiz M et al. Coronary heart disease and periodontitis - a case control study in Chilean adults. J Clin Periodontol 2002; 29:468473.

23. Wahaidi V, Kowolik M, Eckert G, and Galli G. Endotoxemia and the host systemic response during experimental gingivitis. J Clin Periodontol 2011; 38:412-417. 